\title{
Pregnancy-related deaths and complications in women with vascular Ehlers-Danlos syndrome
}

\author{
Mitzi L. Murray, MD, MA'1,2, Melanie Pepin, MS'1, Suzanne Peterson, MD³ and Peter H. Byers, MD ${ }^{1,2}$
}

Purpose: The purpose of this study was to characterize the nature and magnitude of pregnancy risks in women with vascular EhlersDanlos syndrome.

Methods: Pregnancy-related death rate was determined by a review of pedigrees of families with vascular Ehlers-Danlos syndrome. Maternal morbidity was characterized through semistructured interviews with women with vascular Ehlers-Danlos syndrome or their next of kin.

Results: Pregnancy-related deaths occurred in 30 of 565 deliveries (5.3\%). There was no difference in Kaplan-Meier survival curves between parous versus nulliparous women with vascular EhlersDanlos syndrome. Interviews with 39 women indicated that $46 \%$ of deliveries were uncomplicated. The most common pregnancy-related complications were third-/fourth-degree lacerations (20\%) and preterm delivery (19\%). Life-threatening complications occurred in
$14.5 \%$ of deliveries and included arterial dissection/rupture $(9.2 \%)$, uterine rupture $(2.6 \%)$, and surgical complications $(2.6 \%)$. There were 5 maternal deaths in 76 deliveries (6.5\%).

Conclusion: The risk of pregnancy-related complications is increased in women with vascular Ehlers-Danlos syndrome compared with the general population; however, survival data indicate that pregnancy does not appear to affect overall mortality compared with nulliparous women with vascular Ehlers-Danlos syndrome. The data were insufficient to determine whether mode or timing of delivery influenced risk of complications. Women with vascular Ehlers-Danlos syndrome should be engaged in a shared decisionmaking process when contemplating pregnancy and pregnancy management.

Genet Med advance online publication 12 June 2014

Key Words: COL3A1; pregnancy; vascular Ehlers-Danlos syndrome

\section{INTRODUCTION}

Vascular Ehlers-Danlos syndrome (vEDS, EDS type IV) is an autosomal dominant disorder that results from mutations in COL3A1, the gene that encodes the chains of type III procollagen. Complications of this condition arise from diminished tissue integrity and include arterial rupture/dissection and spontaneous bowel perforation. Pregnancy-related complications occur in women with $\mathrm{vEDS}^{1-7}$ and include arterial dissection/rupture, uterine rupture, hemorrhage, premature rupture of membranes, lacerations, and wound dehiscence. The reported risk of maternal mortality per pregnancy ranges from 4.3 to $25 \%,{ }^{1,6,8}$ and few data are available on the magnitude of maternal morbidity. ${ }^{9}$

Currently, there are no guidelines for pregnancy management in women with vEDS, and development of standards for care is challenged by the rarity of the condition and the lack of evidence-based risk information. In general, it is recognized that pregnancies in women with vEDS should be considered high risk and be overseen by a maternal-fetal specialist. Published recommendations include strongly discouraging pregnancy ${ }^{8}$ or encouraging early delivery via cesarean section with or without use of desmopressin to prevent hemorrhage. ${ }^{10,11}$ However, there are few empirical data to support these recommendations, and it is not clear whether pregnancy avoidance benefits overall survival or reduces the risk of serious health complications.

To investigate the magnitude of and circumstances surrounding maternal mortality and morbidity in women with vEDS, we used two approaches: analysis of retrospective pedigree data, and analysis of data collected during semistructured interviews. This empirically derived risk information provides baseline outcome information with which future interventional studies can be compared and will help to guide the development of consensus guidelines on pregnancy management in women with vEDS.

\section{MATERIALS AND METHODS}

\section{Study subjects-retrospective pedigree analysis}

Parous and nulliparous females were identified in pedigrees of families in which the diagnosis of vEDS was confirmed in one or more individuals by biochemical or genetic testing in the Collagen Diagnostic Laboratory, Department of Pathology, University of Washington between 1976 and 2012. A female was included if she had a confirmed diagnosis or was a female relative of the proband with a complication consistent with vEDS (arterial aneurysm, dissection, or rupture; bowel perforation; or organ rupture). These data include pregnancy information on 81 women who were previously reported. ${ }^{1,6}$ The number of

${ }^{1}$ Department of Pathology, University of Washington, Seattle, Washington, USA; ${ }^{2}$ Department of Medicine (Medical Genetics), University of Washington, Seattle, Washington, USA;

${ }^{3}$ Maternal and Fetal Specialty Center, Swedish Medical Center, Seattle, Washington, USA. Correspondence: Mitzi L. Murray (mlmurray@uw.edu) 
deliveries for each woman was determined from the medical records of the proband and other relatives, as available. Those not of reproductive age $(<12$ years) or for whom insufficient information was available were excluded. Pregnancy-related death was defined using the World Health Organization definition of a death occurring during or within 42 days of the end of a pregnancy. ${ }^{12}$

\section{Statistical analysis—retrospective pedigree analysis}

Survival was estimated with the Kaplan-Meier estimator for all female subjects and stratified by parity. Groups were compared using the log-rank (Mantel-Cox) test.

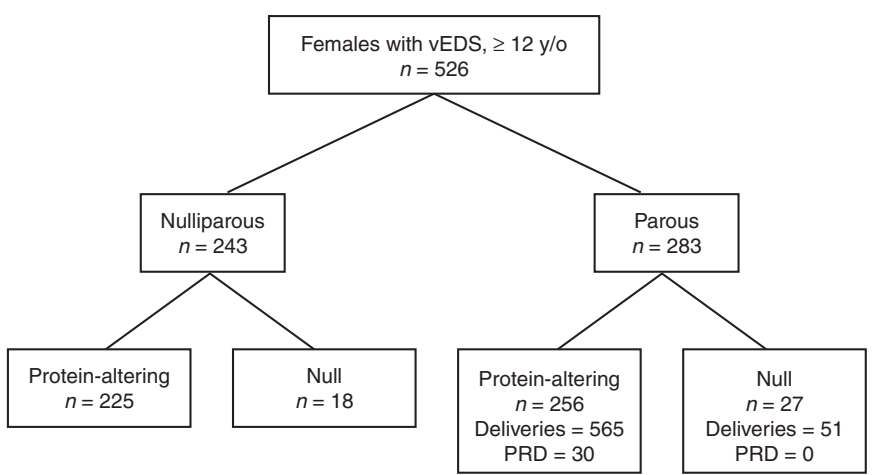

Figure 1 Ascertainment of females with vEDS from a pedigree review, divided by parity and mutation type. PRD, pregnancy-related death; vEDS, vascular Ehlers-Danlos syndrome.

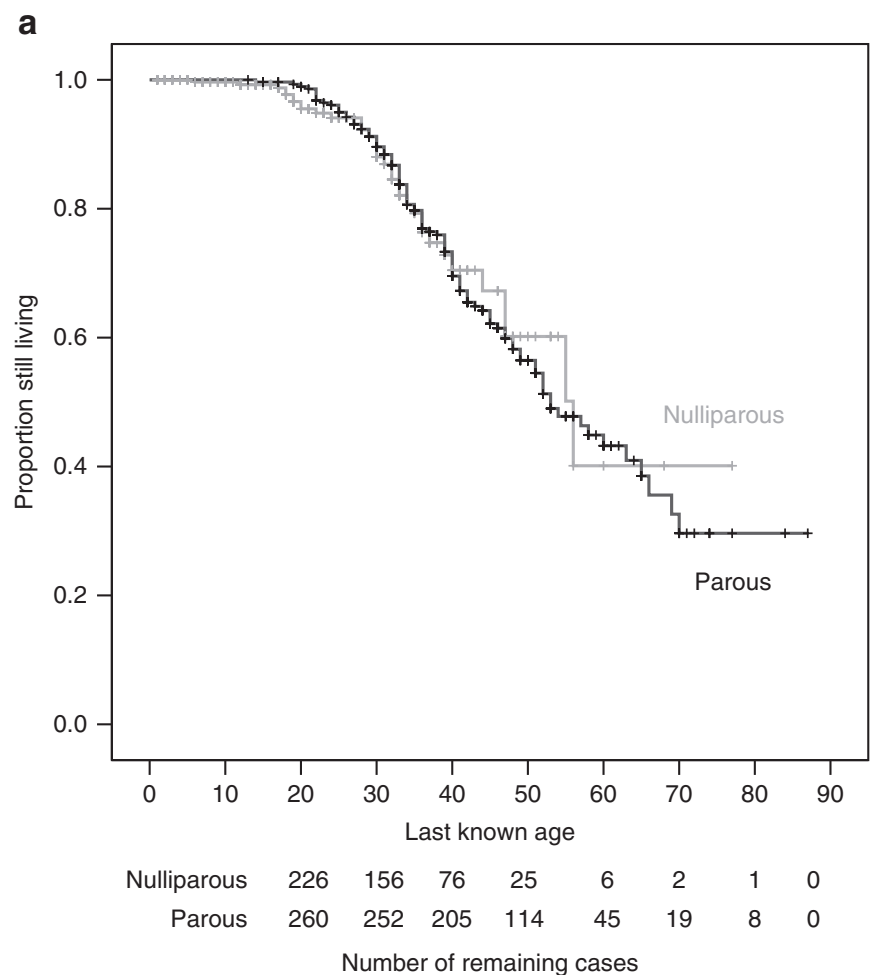

\section{Study subjects-semistructured interview study}

We invited 87 women to participate in an interview study through the Collagen Diagnostic Laboratory, Department of Pathology, University of Washington, through family members who had been tested at this laboratory and through a national support organization (Ehlers-Danlos National Foundation). Eligibility criteria included the presence of a mutation in COL3A1 and self-report of at least one pregnancy, inclusive of all pregnancy outcomes. Next of kin were eligible to participate as proxy if the affected woman had died. Thirty-eight women, representing 113 pregnancies and 82 deliveries, enrolled. Of those who did not participate, 40 did not respond to invitations, 2 declined to participate, and 7 were found to be ineligible because they had not been pregnant. A semistructured questionnaire (available on request) was used to systematically collect diagnostic, birth-related, medical, surgical, obstetric, and family-related histories. We reviewed medical records from five individuals. We defined complications of vEDS to include arterial, intestinal, and uterine ruptures, as well as significant complications during cesarean section. "Other complications" encompassed all other reported pregnancy- or delivery-related events: third- or fourth-degree lacerations, wound dehiscence, hemorrhage sufficient to require blood transfusion or prolonged hospitalization, placenta previa and low-lying placenta, placental abruption, and preterm delivery (including preterm premature rupture of membranes). Pregnancy-related complications were considered to be those that occurred during or within 42 days of the end of a pregnancy. This study was

\section{b}

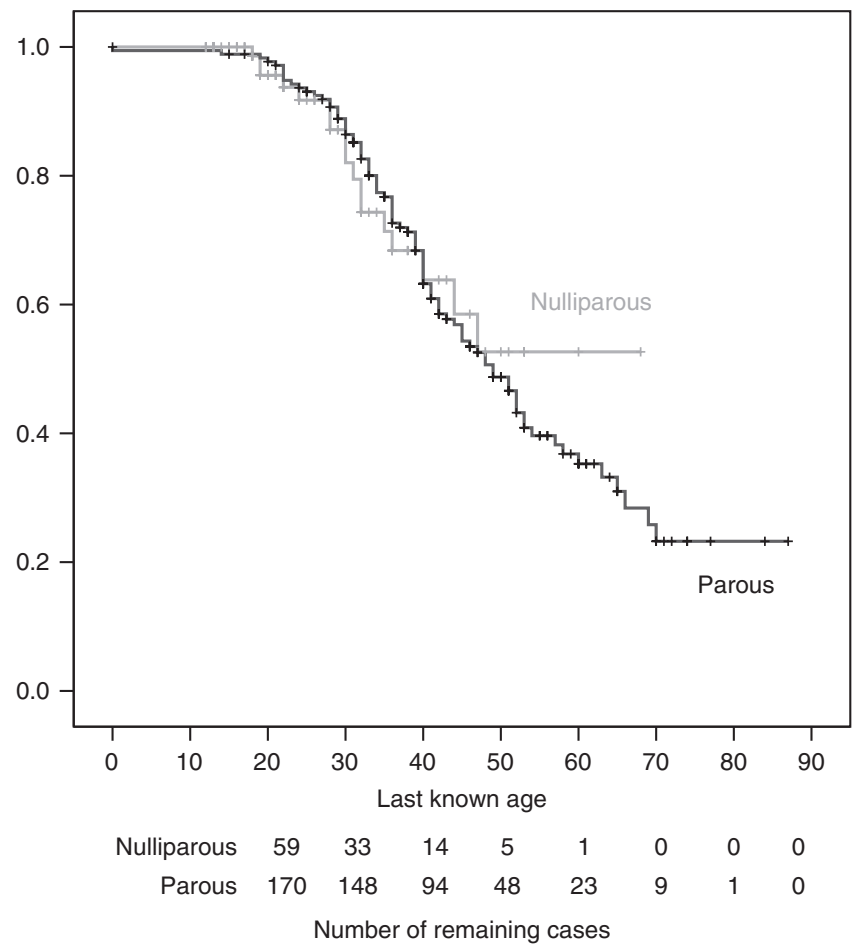

Figure 2 Kaplan-Meier estimates. (a) Overall survival of 526 parous $(n=283)$ and nulliparous $(n=243)$ women of reproductive age with vEDS; (b) overall survival in parous $(n=179)$ and nulliparous $(n=86)$ affected female relatives of probands with vEDS. vEDS, vascular Ehlers-Danlos syndrome. 
approved by the University of Washington Human Subjects Institutional Review Board.

\section{RESULTS}

Retrospective pedigree analysis: pregnancy-related death rate

Review of pedigrees identified 526 women of reproductive age with vEDS (Figure 1). Of these, 243 (46\%) were nulliparous, of whom 157 (64.6\%) were probands. The other 283 (54\%), of whom 104 (37.1\%) were probands, had had at least one pregnancy. In 616 delivered pregnancies, pregnancy-related deaths were reported for 30 women for a calculated pregnancy-related death rate of $4.9 \%$ per delivery. No pregnancy-related deaths were reported for the 27 women (51 pregnancies) with heterozygous null mutations in COL3A1. If those women are removed from the analysis, the pregnancy-related death rate in women with protein-altering mutations is $5.3 \%$ per delivery (30 deaths in 256 women with 565 delivered pregnancies). There were 10 pregnancy-related deaths among the 71 women who had been
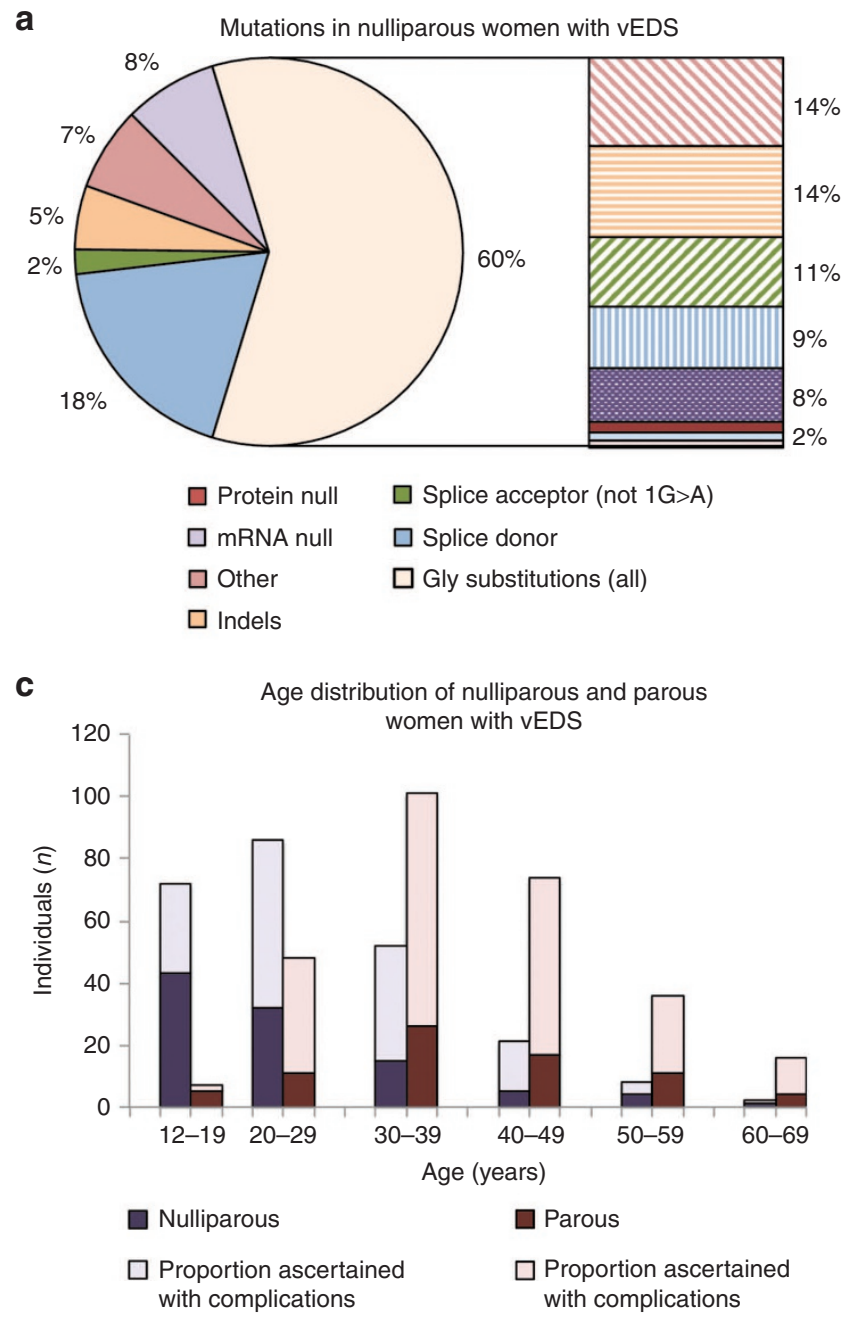

pregnant once, 9 among the 99 who had been pregnant twice, 7 among the 45 who had been pregnant three times, 1 among the 14 who had been pregnant four times, 2 among the 11 who had been pregnant five times, 0 among the 5 who had been pregnant six times, 1 among the five who had been pregnant seven times, and 0 among the three who had been pregnant eight or more times.

\section{Retrospective pedigree analysis: impact of pregnancy on overall survival}

Comparison of nulliparous and parous females with vEDS showed no significant difference in Kaplan-Meier survival curves (Figure 2). The nulliparous and parous groups had a similar distribution of mutation types (Figure 3a,b). Age distribution between the groups was different, with the nulliparous group composed of younger women as compared with the parous group (Figure 3c). The ratio of nulliparous to parous women across reproductive decades of life was similar in the vEDS study population as compared with the general US
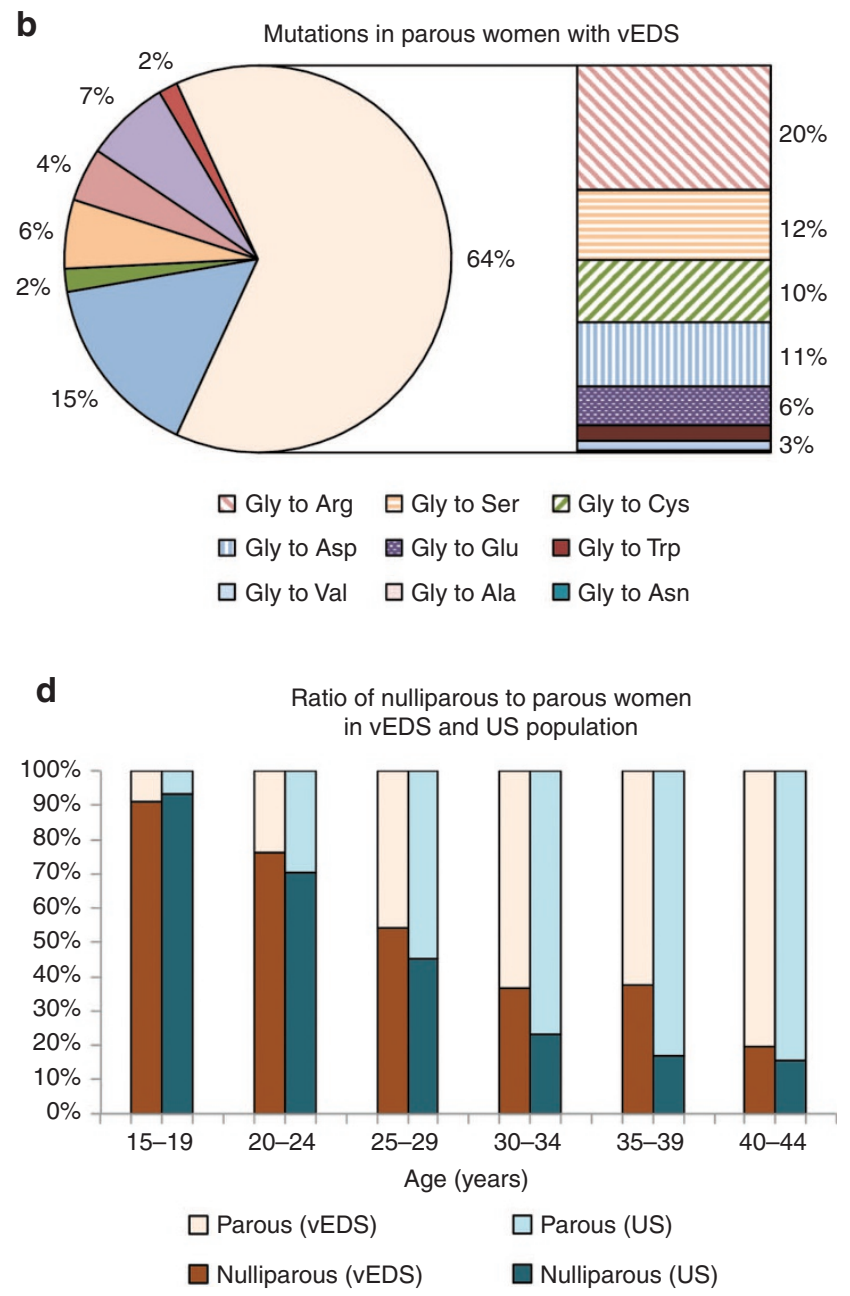

Figure 3 Comparison of nulliparous and parous groups. (a) Distribution of mutation types in the two groups is similar; (b) age distribution difference between groups, with the nulliparous group having a generally younger age distribution as compared with the parous group; (c) proportions of nulliparous and parous women across age groups reflects the proportions seen in the general US population. 
a
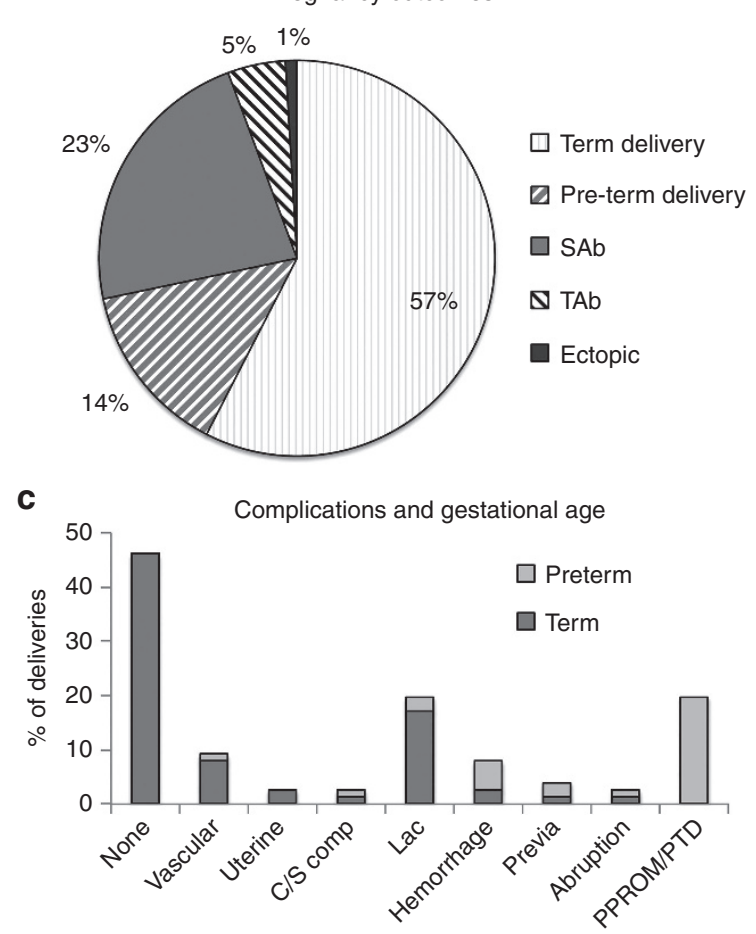

b

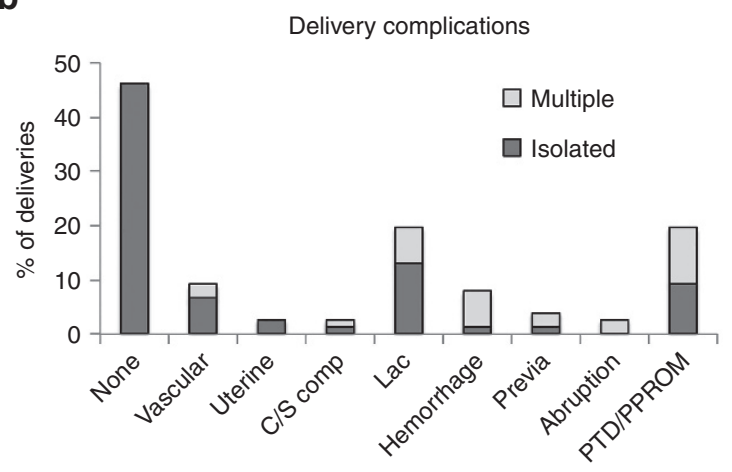

d

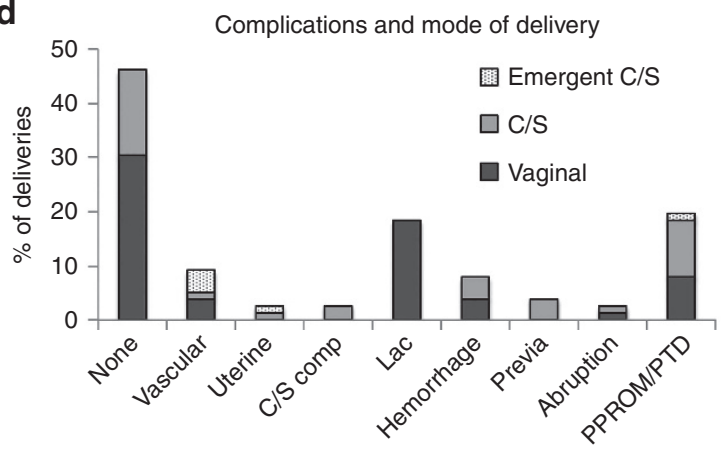

Figure 4 Pregnancy outcomes and complications. (a) Pregnancy outcomes of 113 pregnancies in 38 women with vEDS; (b) delivery complications in 76 deliveries from 35 women with vEDS due to protein-altering mutations; (c) complications in preterm and term deliveries; (d) mode of delivery and complications. Abruption, placental abruption; C/S, cesarean section; Lac, third-/fourth-degree laceration; PPROM, preterm premature rupture of membranes; PTD, preterm delivery; Previa, placenta previa; Sab, spontaneous abortion; Tab, therapeutic abortion; vEDS, vascular Ehlers-Danlos syndrome.

population (Figure 3d), which suggests that the difference in age distributions in our study groups reflects typical reproductive trends with age. As compared with women who had at least one pregnancy, nulliparous women start to accrue vEDS-related complications, although not deaths, at an earlier age (data not shown). To compare the groups of women not diagnosed as a consequence of complications, we compared the Kaplan-Meier survival curves for the parous and nulliparous relatives in each population (Figure $\mathbf{2 b}$ ). Because the nulliparous group is slightly younger, the follow-up is not precisely the same, but the calculated mean age of death is the same in the two groups (53 years).

\section{Interview study: pregnancy complications and maternal morbidity}

Because the clinical details for pregnancies, mode of delivery, and complications provided at the time of testing were often limited, we enrolled 38 women, representing 113 pregnancies and 82 deliveries, in an interview study. Of all pregnancies in this group, $57 \%$ resulted in term deliveries and $14 \%$ in preterm deliveries (Figure 4a). The diagnosis of maternal vEDS was known before conception in 12 pregnancies $(10.6 \%)$, was established during the course of 3 pregnancies $(2.7 \%)$, and was established after completion of 98 pregnancies (86.7\%). Three women (representing seven pregnancies and six deliveries) had null mutations. Those women were excluded from further analysis because of the attenuated phenotype in women with null mutations. Of note, three pregnancy complications were reported in women with null mutations: one had a preterm delivery, and two had third- or fourth-degree vaginal lacerations.

The 35 women with protein-altering mutations in this group represented 76 delivered pregnancies, of which 47 were delivered vaginally $(61.8 \%), 25$ were delivered by routine cesarean section $(32.9 \%)$, and 4 were delivered by emergent cesarean section (5.3\%). No complications occurred in 23 of $47(48.9 \%)$ vaginal deliveries and 12 of $25(48 \%)$ cesarean deliveries (Figure $4 \mathrm{~b}$ ). Nine vEDS complications occurred at term (82\%), and 2 complications occurred preterm (18\%) (Figure 4c). Complications of vEDS included arterial dissection or rupture $(n=7,9.2 \%)$, uterine rupture $(n=2,2.6 \%)$, and complications related to cesarean section $(n=2,2.6 \%)$. The five pregnancy-related maternal deaths resulted from arterial dissection or rupture $(n=4)$ and complications related to dehiscence of a cesarean section incision $(n=1)$. The vascular complications leading to death were two fatal aortic ruptures during labor at term, one fatal aortic rupture 7 days after uncomplicated term cesarean delivery (for breech presentation), and one fatal iliac artery rupture after a fall at 33 weeks gestation. The fifth death resulted from dehiscence after cesarean section (for maternal vEDS and previous cesarean) at 36 weeks gestation. 
The nonfatal vEDS-related complications included one antenatal coronary artery dissection at term, a splenic artery dissection 6 days after term vaginal delivery, multiple arterial dissections in one woman 7 days after term vaginal delivery, two uterine ruptures at term (one antepartum, one intrapartum), and one instance of intraoperative bladder and venous tear during cesarean section (for breech presentation). Other complications included preterm delivery $(n=17,22.4 \%)$, thirdor fourth-degree lacerations ( $n=16,21 \%)$, hemorrhaging sufficient to require transfusion or extended hospital care $(n=8$, $10.5 \%)$, placenta previa or low-lying placenta $(n=3,3.9 \%)$, and placental abruption $(n=2,2.6 \%)$. There were too few events to determine whether mode of delivery affected complication risk, with the exception that third- and fourth-degree lacerations were a feature only of vaginal deliveries (Figure 4d).

\section{DISCUSSION}

Pregnancy in women with vEDS raises several concerns: the recurrence risk for vEDS, the maternal risk for pregnancyrelated death and morbidity, the maternal or fetal risk with prenatal diagnosis, the influence of the mode of delivery on maternal and fetal mortality, and the benefit of surveillance of the maternal vasculature. At this point, only the first question has been answered - the risk that the child will inherit the mutant allele is $50 \%$. The phenotype of the child depends to some extent on the nature of the mutation and its effect on protein production. ${ }^{13}$

A small number of articles have presented primary data that have bearing on the remaining questions and include more than one or two pregnancies. ${ }^{1,6,14}$ The data from Rudd et al., ${ }^{1}$ in which there is probably ascertainment bias, are incorporated into the data provided by Pepin et al. ${ }^{6}$ and into the data we have presented in this article. Most of the other publications present single-case reports of complications and then progressively summarize the data from these reports, ${ }^{3-5,7,8,11,15-19}$ although some small series are appearing. ${ }^{9,20}$ Inevitably, this leads to a view of vEDS and pregnancy that is neither objective nor encouraging. The major conclusion drawn from these studies is that pregnancy uniquely alters the natural history of women with vEDS and leads to early death. None of the previous studies have considered the necessary control group-women with vEDS who have not been pregnant.

In the retrospective pedigree study, we asked whether pregnancy influences life expectancy for women with vEDS. We took advantage of a unique data set at the Collagen Diagnostic Laboratory, University of Washington, which contained information on 572 families with a diagnosis of vEDS confirmed by protein or genetic studies. We counted the number of reported pregnancies for women with vEDS and determined how many deaths were related to pregnancy. When we used these data to compare the Kaplan-Meier survival curves for women who had been pregnant and those who had not, we were surprised to find that the curves overlapped without significant differences in survival. This finding countered the common lore that pregnancy, per se, reduces life expectancy for women with vEDS and that they should be counseled against becoming pregnant or to terminate pregnancy electively if they do become pregnant (see, e.g., ref. 8). Because these findings were unexpected, we looked at ways that differences between nulliparous and parous groups could influence the outcome, regardless of pregnancy. The nulliparous women were younger (as expected from comparison with the US Census data), the ratio of probands to relatives was higher than for the parous group, and the onset of complications in the nulliparous group was earlier-as might be expected from the excess of probands. To try to control for these variables, we compared the women who had been identified because they were relatives of probands and compared the Kaplan-Meier curves for those who had been pregnant and those who had not been pregnant and found that they were similar. Considering this result, it appears that for women who have not had vEDS-related complications before pregnancy, pregnancy itself does not appear to add further risk of death.

This study expands the population of pregnant women reported by Pepin et al. ${ }^{6}$ from 81 to 256 . The pregnancy-related death rate observed in this study is similar to that reported by Pepin et al. (5.3 vs. $4.3 \%){ }^{6}{ }^{6}$ This rate is substantially lower than previous estimates of up to $25 \%$ maternal mortality, ${ }^{1}$ which, we think, are elevated by report or ascertainment bias. Although the per-delivery mortality rate is almost 300 times as high as the pregnancy-related death rate in the general US population of 17.8 per 100,000 live births, ${ }^{21}$ our survival analysis suggests that pregnancy in women with vEDS does not significantly affect overall survival as compared with that of nulliparous women with vEDS. Although pregnancy avoidance can prevent the risk of pregnancy-specific complications such as uterine rupture, almost all deaths that occurred in relation to pregnancy were the result of arterial dissection or rupture, just as they are in nonpregnant women and in men. ${ }^{13}$

There are several limitations to this study that we think are not sufficient to undermine the basic conclusion that pregnancy does not alter the survival of affected women beyond the underlying effects of the disorder. First, because ascertainment was through a clinical diagnostic laboratory, we have identified only individuals in which an event brought the family to medical attention. Second, we included women as affected if they had a biochemical or genetic diagnosis or a confirmed family diagnosis and a history of complications consistent with the diagnosis. We did not include female relatives without a confirmed diagnosis or compatible complications. Third, it is possible that the nulliparous women with vEDS could have a disproportionate burden of early-onset or severe complications that led to advice not to pursue pregnancy. There was a slight excess of women with null mutations in the pregnancy group, and this type of mutation is known to lead to an attenuated phenotype..$^{22}$ When we conducted the analysis with this group excluded, the curves remained similar. The nature of the underlying mutations has an effect on overall complication rate and survival. ${ }^{13}$ There was, however, no difference in the distribution of mutation types between the two groups. Finally, there was a difference in the age distribution between the two groups, with the nulliparous 
group clustering in the younger age groups. This reflects the distribution of ages in the US population between women who have been pregnant and those who have not. In addition, analysis of only relatives ascertained primarily due to family history showed no significant difference in survival when compared by parity. Although these limitations are important to consider, it is not clear that any of these variables substantially alters the conclusion that pregnancy per se does not alter survival.

In our interview study, we characterized pregnancy-related morbidity and tried to determine whether the mode or timing of delivery affects outcome. Most women and their clinicians were not aware of the diagnosis of vEDS before pregnancy ( $>85 \%$ of pregnancies in the group were completed before the genetic diagnosis). Thus, the complication rates we found largely reflect pregnancy-related risks when management is not influenced by awareness of the mother's diagnosis. In this study, almost half of deliveries (vaginal and cesarean) were uncomplicated when managed routinely. We could not be sure whether the mode of delivery affected overall survival because the number of pregnancy-related deaths was too small to allow us to reach a conclusion. The mode of delivery is, however, associated with different morbidity burdens in that the rate of thirdor fourth-degree lacerations (20\%) - a complication solely of vaginal delivery-is close to 10 times the expected rate. ${ }^{23}$ In our interview study, most life-threatening complications occurred in association with term or near-term deliveries; however, the number of these complications is too small to determine whether this represents an increase in risk late in pregnancy or whether this simply reflects the fact that most deliveries in our study occurred at term or near term. Although we had hoped that this study would allow us to reach a clear conclusion with regard to timing and mode of delivery, our data set is too small to determine whether one mode would reduce fatality or complications or both in this setting.

vEDS is a rare disorder, and most clinicians will never encounter a patient in their practice. Close to half of all probands have de novo mutations in COL3A1. Although about half the deliveries in our interview group occurred without complications, knowledge of the diagnosis may facilitate the development of a more comprehensive management protocol, such as ensuring that vascular and general surgeons are on call at the time of delivery. Awareness of the magnitude of risk and the type of complications that may occur feeds the notion that management of pregnancy and delivery in a tertiary care setting is advantageous, although neither our data nor other published studies provide empirical support that this improves outcome.

We think that the data from this study are sufficient to indicate that although there is a risk of life-threatening events and serious complications during pregnancy in women with vEDS, pregnancy itself does not appear to influence longevity. This is a substantial departure from the common view of pregnancy in women with vEDS. Considering this, we suggest that geneticists and obstetricians reconsider previous recommendations in the literature that pregnancy should be actively discouraged in women with vEDS or that termination of pregnancy should be recommended based purely on the woman's genetic diagnosis. For women who wish to carry a pregnancy, preconception consultations (i) with a maternal-fetal specialist to discuss pregnancy and delivery management and (ii) with a geneticist to discuss reproductive options are appropriate. In sum, we recommend that the care team and family formulate a pregnancy management plan that takes into consideration the empirical risk information available, the woman's past medical history and previous complications, and the available health-care resources, including the obstetrician's experience and expertise.

It is clear that further research is needed to investigate whether the mode of delivery or its timing influences the risk of life-threatening complications. We suggest that a multicenter, international study be initiated to answer the remaining pregnancy-related concerns in the context of a wider study of natural history. This could include collaboration between extant clinical centers and academic and commercial testing laboratories to identify families and to review medical histories and records in a comprehensive fashion. Coordination with national peer group organizations could be an important additional recruitment step in this process.

\section{ACKNOWLEDGMENTS}

This study was supported in part with funds from the Freudmann Fund for Ehlers-Danlos Syndrome Translational Research at the University of Washington. We are grateful for the support and participation of many families in this study.

\section{DISCLOSURE}

The authors declare no conflict of interest.

\section{REFERENCES}

1. Rudd NL, Nimrod C, Holbrook KA, Byers PH. Pregnancy complications in type IV Ehlers-Danlos Syndrome. Lancet 1983;1:50-53.

2. Snyder RR, Gilstrap LC, Hauth JC. Ehlers-Danlos syndrome and pregnancy. Obstet Gynecol 1983;61:649-650.

3. Brees CK, Gall SA. Rupture of the external iliac artery during pregnancy: a case of type IV Ehlers-Danlos syndrome. J Ky Med Assoc 1995;93:553-555.

4. Athanassiou AM, Turrentine MA. Myocardial infarction and coronary artery dissection during pregnancy associated with type IV Ehlers-Danlos syndrome. Am J Perinatol 1996;13:181-183.

5. Björck M, Pigg M, Kragsterman B, Bergqvist D. Fatal bleeding following delivery: a manifestation of the vascular type of Ehlers-Danlos' syndrome. Gynecol Obstet Invest 2007;63:173-175.

6. Pepin M, Schwarze U, Superti-Furga A, Byers PH. Clinical and genetic features of Ehlers-Danlos syndrome type IV, the vascular type. N Engl J Med 2000;342: 673-680.

7. Yamashita M, Narita M, Ishihara H, Matsuki A, Oyama T. Uterine rupture in a case with Ehlers-Danlos syndrome type IV-anesthetic considerations. Middle East J Anesthesio/ 1987;9:277-281.

8. Lurie S, Manor M, Hagay ZJ. The threat of type IV Ehlers-Danlos syndrome on maternal well-being during pregnancy: early delivery may make the difference. J Obstet Gynaecol 1998;18:245-248.

9. Dubruc E, Dupuis-Girod S, Khau Van Kien P, et al. [Pregnancy and Ehlers-Danlos vascular syndrome: patients' care and complications]. J Gynecol Obstet Biol Reprod (Paris) 2013;42:159-165.

10. Rochelson B, Caruso R, Davenport D, Kaelber A. The use of prophylactic desmopressin (DDAVP) in labor to prevent hemorrhage in a patient with EhlersDanlos syndrome. NY State J Med 1991;91:268-269.

11. Weinbaum PJ, Cassidy SB, Campbell WA, et al. Pregnancy management and successful outcome of Ehlers-Danlos syndrome type IV. Am J Perinatol 1987;4:134-137. 
12. World Health Organization. Maternal mortality ratio (per 100,000 live births) http://www.who.int/healthinfo/statistics/indmaternalmortality/en/. Accessed 16 March 2014.

13. Pepin M, Schwarze $U$, Leistritz D, Rice KM, Liu M, Byers PH. Survival is affected by mutation type and molecular mechanism in vascular Ehlers-Danlos syndrome (EDS type IV). Genet Med 2014;16:881-888 (this issue).

14. Pope FM, Nicholls AC. Pregnancy and Ehlers-Danlos syndrome type IV. Lancet 1983;1:249-250

15. Erez Y, Ezra Y, Rojansky N. Ehlers-Danlos type IV in pregnancy. A case report and a literature review. Fetal Diagn Ther 2008;23:7-9.

16. Hammond R, Oligbo N. Ehlers Danlos Syndrome Type IV and pregnancy. Arch Gynecol Obstet 2012;285:51-54.

17. Ohkuchi A, Matsubara S, Takahashi K, et al. Ehlers-Danlos type IV in pregnancy with a history of myocardial infarction. J Obstet Gynaecol Res 2009;35: 797-800.
18. Peaceman AM, Cruikshank DP. Ehlers-Danlos syndrome and pregnancy: association of type IV disease with maternal death. Obstet Gynecol 1987;69(3 Pt 2):428-431.

19. Sorokin Y, Johnson MP, Rogowski N, Richardson DA, Evans MI. Obstetric and gynecologic dysfunction in the Ehlers-Danlos syndrome. J Reprod Med 1994;39:281-284.

20. Hurst BS, Lange SS, Kullstam SM, et al. Obstetric and gynecologic challenges in women with Ehlers-Danlos syndrome. Obstet Gynecol 2014;123:506-513.

21. Centers for Disease Control and Prevention CDC CfDC. Accessed 20 May 2013 http://www.cdc.gov/reproductivehealth/maternalinfanthealth/pmss.html.

22. Leistritz DF, Pepin MG, Schwarze U, Byers PH. COL3A1 haploinsufficiency results in a variety of Ehlers-Danlos syndrome type IV with delayed onset of complications and longer life expectancy. Genet Med 2011;13:717-722.

23. Eskandar O, Shet D. Risk factors for $3^{\text {rd }}$ and $4^{\text {th }}$ degree perineal tear. J Obstet Gynaecol 2009;29:119-122. 